Priority Research Paper

\title{
Application of 3D tumoroid systems to define immune and cytotoxic therapeutic responses based on tumoroid and tissue slice culture molecular signatures
}

\author{
Niklas K. Finnberg ${ }^{1}$, Prashanth Gokare ${ }^{1}$, Avital Lev ${ }^{1}$, Sergei I. Grivennikov ${ }^{2}$, \\ Alexander W. MacFarlane IV ${ }^{3}$, Kerry S. Campbell ${ }^{3}$, Ryan M. Winters ${ }^{4}$, Karen \\ Kaputa ${ }^{4}$, Jeffrey M. Farma5, Abbas El-Sayed Abbas ${ }^{6}$, Luigi Grasso7, Nicholas C. \\ Nicolaides ${ }^{7}$ and Wafik S. El-Deiry ${ }^{1}$ \\ ${ }^{1}$ Department of Hematology/Oncology and Molecular Therapeutics Program, Laboratory of Translational Oncology and \\ Experimental Cancer Therapeutics, Fox Chase Cancer Center, Philadelphia, PA, USA \\ ${ }^{2}$ Cancer Prevention and Control Program, Fox Chase Cancer Center, Philadelphia, PA, USA \\ ${ }^{3}$ Division of Basic Science, Fox Chase Cancer Center, Philadelphia, PA, USA \\ ${ }^{4}$ Biosample Repository Facility, Fox Chase Cancer Center, Philadelphia, PA, USA \\ ${ }^{5}$ Division of General Surgery, Department of Surgical Oncology, Fox Chase Cancer Center, Philadelphia, PA, USA \\ ${ }^{6}$ Division of Thoracic Surgery, Department of Surgical Oncology, Fox Chase Cancer Center, Philadelphia, PA, USA \\ ${ }^{7}$ Morphotek Inc., Philadelphia, PA, USA \\ Correspondence to: Wafik S. El-Deiry, email: Wafik.Eldeiry@FCCC.EDU
}

Keywords: 3D, organoid, colorectal cancer, immune cells, biomarkers

$\begin{array}{lll}\text { Received: June 24, } 2017 \quad \text { Accepted: July 20, } 2017 & \text { Published: August 05, } 2017\end{array}$

Copyright: Finnberg et al. This is an open-access article distributed under the terms of the Creative Commons Attribution License 3.0 (CC BY 3.0), which permits unrestricted use, distribution, and reproduction in any medium, provided the original author and source are credited.

\section{ABSTRACT}

We have developed 3D-tumoroids and tumor slice in vitro culture systems from surgical tumor specimens derived from patients with colorectal cancer (CRC) or lung cancer to evaluate immune cell populations infiltrating cultured tissues. The system incorporates patient's peripherally and tumor-derived immune cells into tumoroid in vitro cultures to evaluate the ability of the culture to mimic an immunosuppressive tumor microenvironment (ITM). This system enables analysis of tumor response to standard therapy within weeks of surgical resection. Here we show that tumoroid cultures from a CRC patient are highly sensitive to the thymidylate synthase inhibitor 5-fluorouracil (adrucil) but less sensitive to the combination of nucleoside analog trifluridine and thymidine phosphorylase inhibitor tipiracil (Lonsurf). Moreover, reintroduction of isolated immune cells derived from surrounding and infiltrating tumor tissue as well as CD45+ tumor infiltrating hematopoietic cells displayed prolonged (>10 days) survival in co-culture. Established tumor slice cultures were found to contain both an outer epithelial and inner stromal cell compartment mimicking tumor structure in vivo.

Collectively, these data suggest that, 3D-tumoroid and slice culture assays may provide a feasible in vitro approach to assess efficacy of novel therapeutics in the context of heterogeneous tumor-associated cell types including immune and nontransformed stromal cells. In addition, delineating the impact of therapeutics on immune cells, and cell types involved in therapeutic resistance mechanisms may be possible in general or for patient-specific responses.

\section{INTRODUCTION}

Historically, the successful establishment of exponentially growing viable cells from patient-derived tumors in in vitro cell cultures has been found to occur from a minority of tumor tissues following artificial selection of a sub-population of tumor cells. Such limitations make it difficult to model patient variability in 
drug responses in vitro. Moreover, the lack of stromal cells that play a critical role in tumor viability, growth and drug response in vivo make the prediction of tumor response of immune-based therapeutics challenging. Through recent advances in three-dimensional (3D) culture ("tumoroid") methodologies some of these hurdles are beginning to be addressed. However, it is currently unclear to what extent such models can be engineered to retain important phenotypic properties of infiltrating immune cells from the tumor tissue of origin that mimic the in vivo environment.

A long-standing desire has been to test tumor tissues from patients to rapidly prognosticate treatment outcomes in vitro and develop treatment strategies that account for inter- and intra-patient tumor heterogeneity. Although the methodology to establish tissue in vitro cultures has been available for more than a century [1], the use of two dimensional (2D) culture techniques to establish continuous tumor cell lines from explanted tumor tissue cultures is hampered by artificial culture conditions that severely diminish the success rate, and tumor clonal heterogeneity. Further, time constraints render this approach impractical for clinical application to derive drug response profiles of tumors for specific patients. Recent advances in patient-derived $3 \mathrm{D}$ cultures have facilitated what is perhaps more relevant modeling of cancer in vitro and this can be combined with whole-genome sequencing approaches to couple functional and correlative tumorspecific data with the promise to shape the landscape of precision medicine. 3D in vitro tumoroid cultures can be rapidly established from biopsied or resected tumor tissues and subsequently subjected to in vitro sub-cultivation, expansion and genomic manipulation using e.g. CRISPR/ Cas9 methodology [2]. Patient-specific tumoroid response profiles have been established in relation to their genomic profiles following high-throughput screening against cytotoxic drug libraries $[3,4]$. Clinical trials are currently ongoing to determine whether this approach can prognosticate treatment outcome of pancreatic, liver and colorectal cancer patients $[5,6]$. Interestingly, tumoroid cultures also maintain the tumor heterogeneity of the tumor-of-origin and since normal surrounding tissues can be established in addition with little modification to the culture media, a patient disease-specific genomic or proteomic tumor profile can be established in relation to the normal tissue from which the tumor arose $[5,7]$. Tumoroid cultures can also be implanted orthotopically in immunodeficient mice to address the impact of diseasespecific driver genes and their impact on the tumoroid's capacity to metastasize to distant organs [8].

Although tumoroid 3D cultures allow the expansion of the tumor epithelium they are typically devoid of nonepithelial stromal cells from the tumor-of-origin and therefore their use is limited in addressing therapeutic strategies targeting this compartment. The ability to retain tumor specimens with stroma is important to establish models that can represent in vivo tumor biology with a microenvironment including stromal and immune cells that play a prominent role in modulating processes such as tumor progression and immune evasion [9-12]. Alternative in vitro culturing methodologies have been employed to maintain the heterogeneous tumor microenvironment by embedding tissue fragments in a $3 \mathrm{D}$ culture matrix in an air-liquid interface culture system (ALI) to maintain a polarized epithelial cell layer in close proximity to stromal cells. This culture system has been shown to yield expanding cystic structures from neonatal small intestine for up to 350 days in culture [13]. The wall of the intestinal cysts consists of a polarized epithelial monolayer with an apical inner luminal surface and a basal outer surface in close proximity to alpha-smooth muscle actin expressing myofibroblasts containing fully differentiated microstructures of cultured intestinal epithelial cells when expanded in collagen [13]. Furthermore, this ALI model system may be used for oncogenic in vitro transformation of primary epithelial and mesenchymal explanted colon, stomach and pancreatic mouse tissues from mice [14].

Here we have optimized a novel 3D culture methodology to investigate and characterize resected tumor tissue in the presence of stromal and immune cells. This system can maintain primary tumor cells and an associated tumor microenvironment for up to forty-four (44) days following isolation. Importantly, these cultures maintain expression of the soluble tumor markers CA19-9 and CEA similar to those of the primary tumor in patients. Application of this system enabled immune profiling of blood and tumor tissue that found patients with reduced numbers of NK cells and increased presence of immunerelated myeloid-derived suppressor cells (MDSC) in their tumors, indicating a potential immunosuppressive profile. Interestingly, we observe reduced levels of CD3 expressing cells in our slice culture despite retention of CD45+ cells. This primary culture model system may be useful for identifying in vitro drug response as well as biomarker assessment to help guide personalized therapy and molecular profiles to define predicative molecular profiles of drug responsive tumors.

\section{RESULTS}

\section{Establishment of tumoroid air-liquid interface (ALI) 3D cultures and tumoroids}

We established 3D tumoroid cultures from resected tumor tissues of patients who provided their informed consent and underwent surgery at Fox Chase Cancer Center (Table 1). There were four (4) tissue specimens of colorectal cancer isolated from various segments; two (2) specimens were obtained from the colon and two (2) specimens were obtained from the rectum. We were able to establish both 3D ALI cultures and epithelial tumoroid 
cultures from three (3) out of four (4) of the tissues that were provided (Table 1). In one case (ReCa030917), we were also able to establish organoid cultures from adjacent normal mucosa. Upon troubleshooting the case ( $\mathrm{ReCa}$ 091516) from which we failed to prepare tumoroid and ALI cultures it was discovered that the specimen that was processed only contained fibrous stroma and was completely devoid of tumor epithelium. A similar situation was also present in the case of the pancreatic cancer specimen (Table 1) where we obtained $<0.5 \mathrm{~cm}^{3}$ of tumor tissue. Upon histological inspection of $\mathrm{H} / \mathrm{E}$ stained tissues, approximately $10-15 \%$ of the tissue-of-origin used for cell culturing contained tumor epithelium with the rest of the tissue specimen containing tumor stroma - a common feature of pancreatic cancers. Although, this did not prevent us from establishing 3D ALI cultures it had a negative effect on establishing epithelial tumoroid cultures that could be subjected to sub-passaging. Tumor tissues were also obtained from three (3) non-small cell lung cancer (NSCLC) patients who underwent surgical resection. These specimens included two (2) squamous NSCLC tissues and one (1) adenocarcinoma NSCLC tissue. We successfully established 3D epithelial tumoroids and ALI cultures from all the lung cancer specimens. However, the squamous NSCLC tumoroids (isolated from two different patients) propagated poorly under our in vitro culture conditions and underwent senescence after two (2) passages. By contrast, we were able to propagate tumoroids established from the lung adenocarcinoma (NSCLC) case for eight (8) passages. These lung adenocarcinomas tumoroid cultures were also subjected to drug screening experiments.

\section{Growth factor-dependent selection of CRC 3D cultures enrich for tumoroids expressing established tumor markers}

The epithelial CRC tumoroid cultures were established from multicellular aggregates in matrigel and grown according to what has previously been described [15] (Figure 1A). In order to promote the selection of cancer cells, WNT3A was excluded from the chemically defined WENRAS media as previously described [3]. One of the CRC tumoroid specimens was relatively insensitive to growth factor deprivation of the WENRAS media including depletion of noggin, EGF, WNT3A and rspo1/ WNT3A as determined by calcein uptake (Figure 1B). However, the CRC tumoroids were highly sensitive to treatment with doses of the MDM2 inhibitor nutlin3A to promote selection of CRC tumoroids with mutant Tp53 [16] (Figure 1B). Indeed, this CRC tumoroid expressed the tumor marker EpCam, beta-catenin and nuclear levels of p53 in the absence of p53-triggering stimuli in culture in vitro (Figure 1C-1D). Our data reinforce prior published findings that tumoroids expressing tumor-specific markers

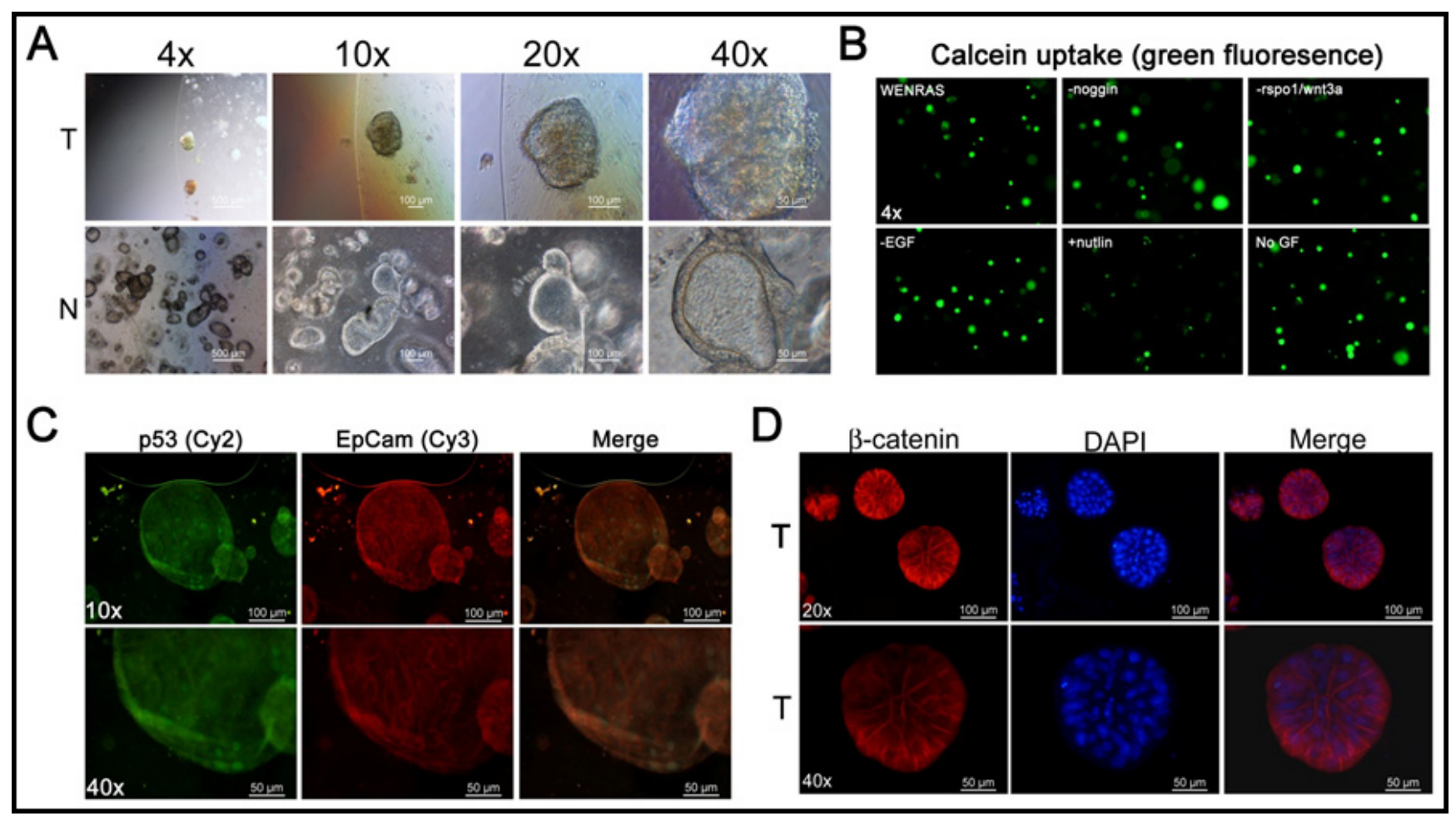

Figure 1: 3D colon cancer organoid cultures with growth in vitro and expression of tumor-associated markers. A. Bright field imaging of 3D organoid cultures of normal colon $(\mathrm{N})$ and colorectal cancer (CRC) (T). B. Calcein uptake in CRC tumoroid culture subjected to selection using growth factor deprivation. Immunofluorescence for tumor markers p53, EpCam and beta-catenin C., D. 
can be quickly established from small amounts of resected tumor specimens.

\section{ALI cultures express the tumor markers CA19-9 and CEA}

To further investigate the expression of tumor markers in 3D tumoroid cultures we chose to analyze the expression of carbohydrate antigen 19-9 (CA19-9) and the carcinoembryonic antigen (CEA) tumor shed antigens (TSAs) commonly used clinically as cancer biomarkers [17-19]. In order to verify tumor-specific expression of these biomarkers, we performed immunohistochemical (IHC) staining of the tumor-of-origin and respective ALI 3D tumor culture (Figure 2A and 2B). Although, the NSCLC culture stained positive for CEA (both squamous cell and adenocarcinoma) they were devoid of staining for CA19-9 (Figure 2A). By contrast, the CRC ALI cultures expressed high levels of both CEA and CA19-9 (Figure 2B). We investigated whether these cultures also shed the biomarkers in the media after prolonged culture and how the levels compare to the levels present in the serum of the patient from which these tumor tissues originated. In order to address this question, we determined the levels of CEA and CA19-9 using ELISA (Figure 2C and 2D). Indeed, as predicted by IHC, 3D ALI tumor cultures with high expression of CA19-9 and/or CEA also shed readily detectable amounts of these biomarkers in their media up to forty-four (44) days after establishing the ALI in vitro cultures. From this followed that only cultures from the pancreatic tumor and CRC expressed high levels of CA199 in the media whereas 3D ALI cultures of NSCLC did not shed CA19-9 in the media nor was this biomarker abnormally elevated in the serum of such patients (Figure $2 \mathrm{C}$ and 2D). Collectively, our data suggest that tumor $3 \mathrm{D}$ ALI cultures can retain the expression pattern of clinically relevant tumor biomarkers that are present within the tumor-of-origin for an extended period of time in culture.

\section{Immune cell profiling of tumors and patient blood reveal differential infiltration of immune cells in donor tissues}

A critically important component of the tumor microenvironment is the presence of immune cells that can promote or inhibit tumor progression. In order to address to what extent our organoid 3D ALI cultures contained immune cells with potential importance for

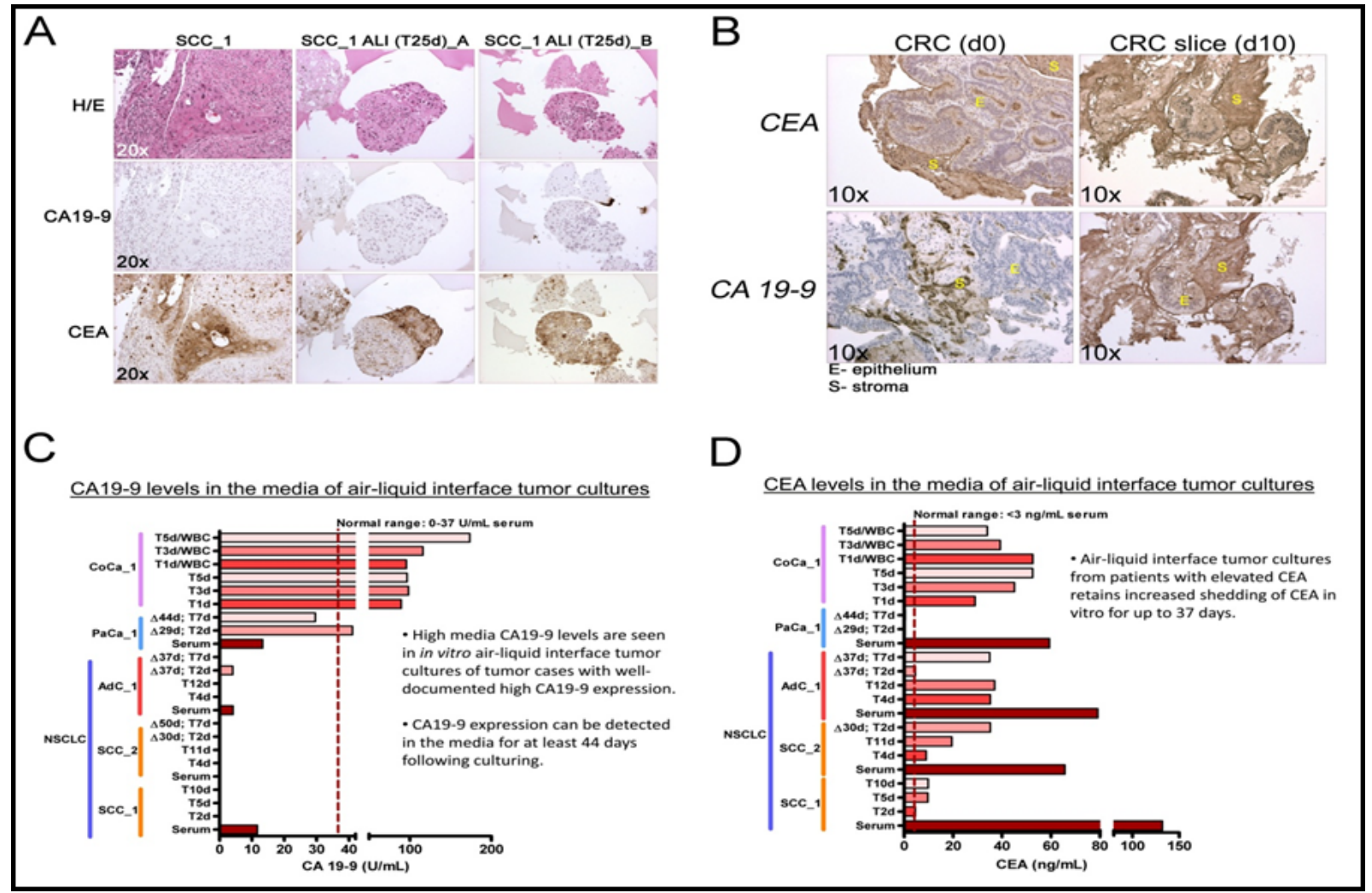

Figure 2: 3D air-liquid interface (ALI) lung cancer in vitro cultures express several tumor serum markers in culture over extended culture periods. A., B. IHC for CA19-9 and CEA expression in squamous cell carcinoma and CRC tumor-of-origin and in ALI tumor cultures. C., D. Assessment of CEA and CA19-9 in patient serum and 3D culture media over time. Squamous cell carcinoma (SCC) ALI. T1-44d - days in culture following isolation. $\Delta 29 \mathrm{~d}-29$ days before sampling. 
Table 1: Tumor-of-origin for establishing 3D tumoroid ALI cultures.

\begin{tabular}{|c|c|c|c|}
\hline ID & Anatomical site & Pathology evaluation & Remarks \\
\hline $\mathrm{CoCa} 091516$ & Rectum & Rectal cancer & $\begin{array}{l}\text { Specimen did not yield growing organoid } \\
\text { culture, histopathological evaluation of } \\
\text { the provided tissue specimen showed only } \\
\text { connective tissue. }\end{array}$ \\
\hline CoCa100316 & Colon & Colon adenocarcinoma & $\begin{array}{l}\text { Organoids and slices were established for short- } \\
\text { term culture }\end{array}$ \\
\hline $\mathrm{CoCa} 122316$ & Colon & Colon adenocarcioma & $\begin{array}{l}\text { Organoids and slices were established for short- } \\
\text { term culture }\end{array}$ \\
\hline LuCa121516 & Lung & $\begin{array}{l}\text { Squamous cell carcinoma (SCC), grade } \\
\text { II }\end{array}$ & $\begin{array}{l}\text { Organoids and slices were established for short- } \\
\text { term culture. Slow growing organoids. }\end{array}$ \\
\hline LuCa122716 & $\begin{array}{ll}\text { Lung, } & \text { right } \\
\text { upper } & \text { lobe, } \\
\text { lobectomy } & \end{array}$ & $\begin{array}{l}\text { Adenocarcinoma, Invasive moderately } \\
\text { differentiated lung adenocarcinoma, } \\
\text { WHO mixed subtype, papillary } 70 \% \text {, } \\
\text { micropapillary } 20 \% \text {, and acinar } 10 \% \text {. }\end{array}$ & $\begin{array}{l}\text { Organoids and slices were established for short- } \\
\text { term culture. ALI culture show epithelial growth. } \\
\text { Epithelial organoids has been subpassaged. }\end{array}$ \\
\hline LuCa010517 & $\begin{array}{l}\text { Lung, left upper } \\
\text { lobe, lobectomy }\end{array}$ & $\begin{array}{l}\text { Metastatic Squamous Cell Carcinoma } \\
\text { (SCC), grade II }\end{array}$ & $\begin{array}{l}\text { Organoids and slices were established for short- } \\
\text { term culture. Slow growing organoids. }\end{array}$ \\
\hline $\mathrm{PaCa} 022417$ & Pancreas & Adenocarcinoma & $\begin{array}{l}\text { Low tumor yield, specimen contained mostly } \\
\text { connective tissue. }\end{array}$ \\
\hline $\mathrm{ReCa030917}$ & Rectum & Rectal adenocarcinoma & $\begin{array}{l}\text { Tumor tissue content sufficient to establish } \\
\text { organoid and 3D cultures }\end{array}$ \\
\hline ChCa031017 & Liver & Cholangiocarcinoma & $\begin{array}{l}\text { Three-dimensional ALI cultures established and } \\
\text { 3D early passage organoid culture. }\end{array}$ \\
\hline
\end{tabular}

tumor etiology or the tumor response to immune oncology drugs we performed immune profiling on blood and tumor tissue from patients from which we established 3D ALI in vitro cultures (Figure 3). Data from one patient with lung adenocarcinomas indicated an increased number of myeloid-derived suppressor cells (MDSCs; CD206+/ CD33+/HLA-DR-) and double negative (CD4-/CD8-) $\mathrm{T}$ cells infiltrating this tumor when compared to the corresponding numbers of these cells in the blood (Table
2). By contrast, the number of tumor infiltrating NK cells and monocytes were significantly reduced within the tumor as compared to the numbers in blood. Taken together this may indicate immune suppression of the tumor-of-origin suggesting that correlating the immune profiles of patients with the donating tumor tissue could yield important information with respect to subsequent analysis of the tumor tissues in the 3D ALI cultures. To address potential differences in longevity of immune cells

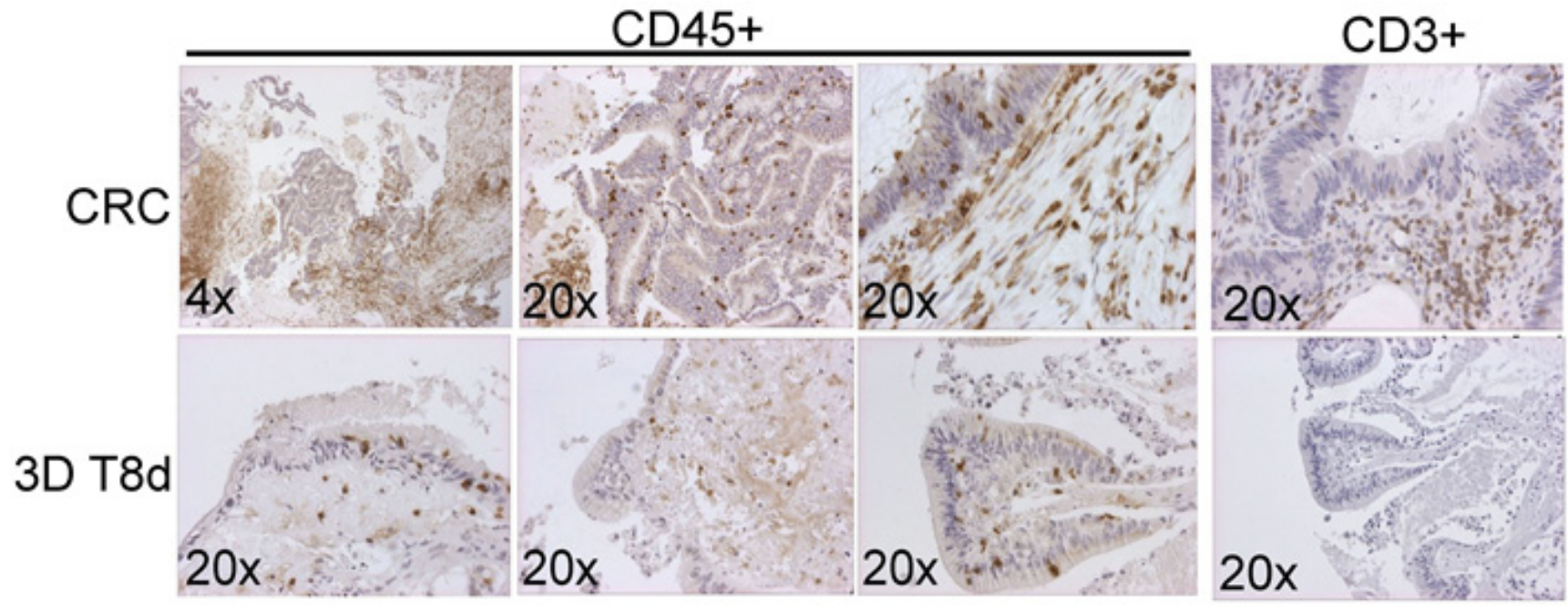

Figure 3: Immune profiling of patient donor tissues and the expression of immune cell markers from 3D ALI cultures in vitro. $\mathrm{IHC}$ for $\mathrm{CD} 45+$ and $\mathrm{CD} 3+$ cells in a $\mathrm{CRC}$ and the corresponding $3 \mathrm{D}$ organoid at eight (8) days after initial culture. 
Table 2: Immune cells in blood and tumor specimen used to establish 3D cultures.

\begin{tabular}{|l|c|c|}
\hline \multicolumn{1}{|c|}{ Immune cells } & LuCa122716 (NSCLC, adenocarcinomas) \\
\hline Myeloid cells (CD45+/CD206+)a & Blood (\%) & Tumor (\%) \\
\hline MDSC (CD33+/HLA-DR-) & & $1.20^{\mathrm{a}}$ \\
\hline Monocytes, CD16+(CD14+/CD16+) & $0.41^{\mathrm{a}}$ & $0.32^{\mathrm{a}}$ \\
\hline Monocytes, CD16- (CD14+/CD16-) & $19.7^{\mathrm{a}}$ & $1.98^{\mathrm{a}}$ \\
\hline T cell(CD45+/CD3+)b & $47.7^{\mathrm{a}}$ & $29.9^{\mathrm{b}}$ \\
\hline T-cell, CD4+ & & $50.0^{\mathrm{b}}$ \\
\hline T-cells, CD8+ & $30.0^{\mathrm{b}}$ & $6.83^{\mathrm{b}}$ \\
\hline T-cells, CD4-/CD8- & $42.2^{\mathrm{b}}$ & $13.2^{\mathrm{b}}$ \\
\hline T-cells, CD4+/CD8+ & $17.7^{\mathrm{b}}$ & \\
\hline NK cell (CD45+/CD56+)c & $10.0^{\mathrm{b}}$ & $2.07^{\mathrm{c}}$ \\
\hline Immature (CD117+/CD94-) & & $19.7^{\mathrm{c}}$ \\
\hline CD16+/CD328+ & $0.075^{\mathrm{c}}$ & \\
\hline
\end{tabular}

aPercent of Myeloid cells (CD45+/CD206+)

bPercent of $\mathrm{T}$ cell population (CD45+/CD3+)

'Percent of NK cells (CD45+/CD56+)

in our 3D ALI cultures we performed IHC on the cultured tissues (Figure 3). Interestingly, in our CRC in vitro cultures we observed the frequent presence of CD45+ cells in the tumor-of-origin. Indeed, CD45+ cells were also present in the cultured tissue following eight (8) days of in vitro 3D culture (Figure 3 ). By contrast, we observed a marked drop in the number of CD3+ cells as compared to the tumor-of-origin following the same length of culturing. This would suggest that although CD45+ hematopoietic cells are present in both the tumor-of-origin and the 3D cultures of CRC tissue, there is a marked reduction in the number of $\mathrm{CD} 3+$ cells under these culture conditions.

\section{Evaluation of cytotoxic responses in cancer 3D organoid cultures reveals differential responses to FDA-approved drugs}

To investigate drug responses in the 3D cultures, we established 3D tumoroids from a resected colon adenocarcinoma (Figure 4A) and cultured these in the absence of WNT3A as previously shown (Figure 1B). These cultures retained some of the cytological hallmarks of the tumor-of-origin as evident by H/E staining (Figure 4C). Next, we subjected these tumoroid cultures to screening using FDA-approved compounds commonly used to treat colorectal cancer. We also included the MEKinhibitor trametinib which is not yet approved for CRC therapy but has shown promising single-agent activity in a previously published drug screen of CRC tumoroids [3] (Figure 4B). After five (5) days of culture we found that CRC patient tumoroids are particularly sensitive to treatment with the approved drugs 5 -fluoruracil (5-FU) and trametinib. Furthermore, regorafenib, cetuximab and irinotecan (CPT-11) in descending order provided similar dose-response profiles but with higher $\mathrm{IC}_{50}$ values as compared to 5-FU. Oxaliplatin and TAS-102 (trifluridine/ tipiracil) did not yield interpretable dose-response profiles for the drug concentrations that were tested. Our data indicates that $3 \mathrm{D}$ tumoroid in vitro cultures can be interrogated in a high-throughput format using cytotoxic drugs within weeks following surgery.

\section{DISCUSSION}

Recent advances in 3D tumoroid culture methodology have enabled scientists to replicate the complex tumor organ system composed of multiple cell lineages including tumor epithelium, stromal cells and vasculature in vitro to better study the complex interaction of these cells in supporting tumor growth and potential drug response. Both the normal and tumor epithelium can now be readily cultured from biopsies, propagated and expanded in vitro using defined culture conditions. Advances in generating tumoroids from pancreatic cancer [20], glioblastoma [21], prostate cancer [22, 23] and colorectal cancer [3] reveal that patient-derived tumoroids recapitulate patient-specific histological features. These tumoroids can also be cryopreserved in a similar manner as continuous cell lines and can subsequently be characterized by genomics, transcriptomics and using high-throughput in vitro drug screening assays, something that has spurred initiatives to develop live patient-derived tumoroid biobanks [1]. It is possible that data from tumoroid drug screening assays not only will complement and corroborate tumor genomic data but may in addition provide prediction on drug responses where sequencing data alone might fall short. Unfortunately, at this time the tumoroid systems are limited in their capacity to predict responses to novel promising immune oncology drugs since they promote the expansion of the tumor epithelium 
and do not support the maintenance and expansion of patient-specific immune and stromal cells that contribute to an immune suppressive tumor microenvironment.

Here we show that 3D ALI tumoroid cultures that include a patient-specific stromal component can be maintained from resected solid tumor specimens when cultured in a Matrigel matrix for up to forty-four (44) days as determined by the expression of TSA biomarkers CEA and CA19-9 in the media. Previous studies on mouse neonatal small intestinal specimens indicate that tissue can be preserved for at least 350 days when isolated from young mice using a similar system [13]. The longevity estimate here would suggest that the living tumor cells are being preserved in our system and it may be possible to extend the life of the cell population. Thus, our finding might be less surprising considering the previous discoveries. However, it remains to be addressed to what extent the different cell populations are maintained in the stroma when compared to the donating tumor-of-origin. For example, although we readily detect CD45+ cells in the 3D ALI cultures, a clear reduction in the presence of CD3+ cells in the tissues was observed following eight (8) days in culture. Moreover, we were only able to document outgrowth of tumor-associated fibroblasts in one of our squamous 3D ALI NSCLC cultures suggesting that the culture conditions did not support the long-term expansion of the stromal compartment. The above might be less surprising since the chemicallydefined WENRAS media employed in this study do not contain growth factors that can support for myeloid and lymphocytic cells, including FGF and interleukins. In fact, one component of the WENRAS media is the signaling peptide noggin which acts as an inhibitor of the bone morphogenic protein-4 (BMP-4), a member of the transforming growth factor-beta (TGF-beta) superfamily [24]. BMP signaling limits hyper-proliferation of the intestinal epithelium and maintains differentiation along the crypt-villus axis by forming a gradient of BMPactivity where mesenchymal cells beneath the crypts express high levels of noggin whereas high levels of BMP-2 and-4 are expressed by mesenchymal cells both in the crypt and the villi [25-28]. Recent evidence has also shown that BMP-signaling constrains the selfrenewal of Lgr5+ cells via SMAD-mediated repression

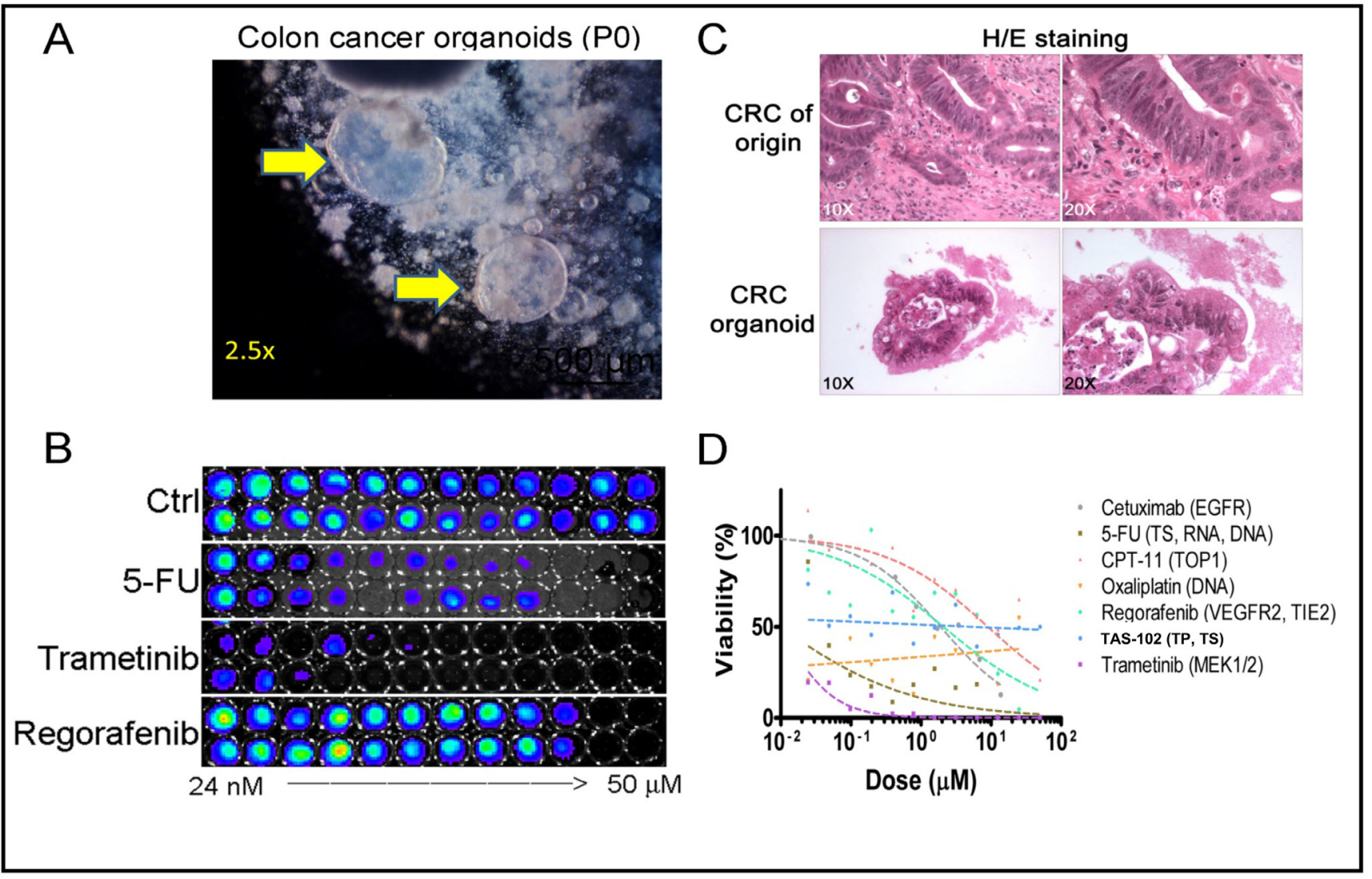

Figure 4: Assessment of CRC patient tumoroid responses to cytotoxic drugs in vitro at 2 weeks following tumor resection. A. Bright field imaging of 3D organoid cultures from a colorectal cancer (CRC). B. CellTiter-Glo (ATP content) was used to determine the dose-response characteristics of a CRC tumoroid following treatment with FDA-approved drugs and trametinib. A duplicate 12-point dose titer was used for each drug that was investigated with 5-10 organoids per 96-well. C. H/E staining of the CRC of origin and tumor organoid cultures. D. Dose-response relationship of FDA- approved drugs and trametinib in CRC tumoroid cultures. 
of the stem cell signature [29]. SMAD4 expression is frequently lost in invasive colorectal cancer, indicating that the quenching of the BMP-signaling pathway in CRC tumoroids through noggin supplementation may be less essential for successful tumoroid in vitro culture [30, 31]. Indeed, one of our CRC tumoroid cultures was completely refractory to the withdrawal of recombinant noggin from the WENRAS media. Thus, further optimization of the growth factors that constitute the WENRAS media is likely necessary to support the diverse cell types that constitute the tumor stroma.

In conclusion, tumor 3D ALI cultures retain the capacity to maintain tumor stroma and characteristics of the primary tumor including the long-term production of CEA and CA19-9 TSAs ( $>44$ days) following isolation. CA19-9 and CEA are commonly used to monitor CRC in the clinic. In our system, the pancreatic cancer specimens expressed high levels of CA19-9 consistent with this being a biomarker often used to detect and monitor treatment of patients with pancreatic cancer. Profiling of immune cell content in the blood and tumors from patients have identified varying populations of myeloid-derived suppressor cells (MDSC), double-negative T cells (DNT), mature NK cells, and monocytes across each tumoroid that appear to correlate with growth and drug response. The fact that these cultures maintain the production of TSAs makes tumoroids useful to better understand the effects TSAs have on tumor immune suppression. Recently, Kline et al. have shown that the CA125 TSA is immunosuppressive to antibody-based tumor therapies [32]. This system will enable better understanding of how the alternative immunosuppressive mechanism can affect the growth and stasis of tumors in the presence of of a given patient's immune system. Additional studies are being designed to better understand the relationship of these cell subtypes and response to therapeutics with the immune microenvironment within the tumoroid cell culture.

\section{MATERIALS AND METHODS}

\section{Tissue isolation and blood collection}

Surgically resected tumor tissues were obtained from eleven (11) patients subjected to treatment at Fox Chase Cancer Center (FCCC). Patient material was collected from a total of five (5) colorectal cancer cases, three (3) NSCLC cancer cases, two (2) cases of pancreatic cancer and one (1) case of cholangiocarcinoma. Tissue fragments representative of what was used for in vitro culture were sampled and fixed in formalin overnight. The remaining tissues were chopped into approximately 5-mm pieces and washed with cold PBS as previously described $[15,33]$. Blood was collected in K2-EDTA blood collection tubes (BD Vacutainer Lavender) and serum blood collection tube (BD Vacutainer Red Top) from each patient immediately prior to surgery. Blood was subjected to $\mathrm{RBC}$ lysis solution (BioLegend) to remove red blood cells and washed in ice cold $\mathrm{PBS} / 2 \% \mathrm{FCS}$. The remaining WBCs were stored at $4^{\circ} \mathrm{C}$ in Streck Cell Preservative ${ }^{\mathrm{TM}}$ (Thermo Fisher Scientific) and subsequently analyzed by flow cytometry. Blood was also allowed to clot and the serum was separated by centrifugation for 10 minutes at $3,500 \mathrm{rpm}$, aliquoted and stored at $-80^{\circ} \mathrm{C}$. All experiments with patient tissues were performed according an approved protocol from the Institutional Review Board of FCCC and all patient tissue or blood samples were obtained with informed consent.

\section{Establishment of 3D air-liquid interface (ALI) \& tumoroid cultures}

ALI cultures were generated in a similar manner as previously described [13]. Cell culture inserts (MillicellCM, Millipore) for 24-well plates were coated with Matrigel (BD bioscience) to generate an acellular bottom layer. The Matrigel was allowed to solidify for 10 minutes at $37^{\circ} \mathrm{C}$. The tumor tissue fragments were washed in icecold PBS and minced rapidly by using iris scissors on a cold tissue culture plate to prevent drying of the tissue. Approximately $30-50 \mathrm{mg}$ of minced tissue was mixed with $400 \mu \mathrm{L}$ of ice-cold Matrigel by pipetting using a $2.0 \mathrm{ml}$ pipette and transfer to the cell culture insert. The tissue was allowed to solidify for 10 minutes at $37^{\circ} \mathrm{C}$. The outer dish was subsequently filled to cover the acellular layer with WENRAS media [14, 30] (advanced Dulbecco's modified Eagle medium/F12 [Invitrogen] supplemented with penicillin/streptomycin [Invitrogen], $10 \mathrm{mmol} / \mathrm{L}$ HEPES [Invitrogen], Glutamax [Invitrogen], 1 x B27 [Invitrogen], $1 \mathrm{mmol} / \mathrm{L} \quad \mathrm{N}$-acetylcysteine [Sigma] containing the following growth factors: gastrin, nicotinamide, EGF, recombinant noggin, WNT3Aand RSPO1-conditioned media. The media was also supplemented with A83-01, SB202190 and Y-27632 as described previously [15]. Tumoroid cultures were prepared by washing the tissue fragments 6-8 times in 10 $\mathrm{mL}$ of ice-cold PBS allowing the tissue to settle after each wash and removing the supernatant. The fragments were incubated in digestion buffer containing collagenase IV and dispase II for approximately one (1) hour at $37^{\circ} \mathrm{C}$. The supernatant was collected in a $15-\mathrm{ml}$ tube and centrifuged for 3 minutes at $200 \mathrm{x}$ g. The supernatant was collected and tumor infiltrating immune cells were enriched by using Percoll separation as previously as described [34] for immune cell profiling (see below). The supernatant was washed in PBS once, the pellet was dissolved and the number of tissue fragments were counted, seeded in $40 \mu 1$ of Matrigel at a density of 25-40 tumoroids/24-well and cultured in WENRAS medium. The WENRAS media 
was changed every other day and the tumoroids were passaged at a 1:2 - 1:6 ratio approximately once every week as previously described [15] using TrypLExpress (Invitrogen) supplemented with Y-27632 (Sigma-Aldrich).

Drugs used in this study were obtained from the following sources: Cetuximab (Fox Chase Cancer Center Pharmacy), 5-FU (Fox Chase Cancer Center Pharmacy), CPT-11 (Fox Chase Cancer Center Pharmacy), oxaliplatin (Fox Chase Cancer Center Pharmacy), regorafenib (MedKoo, catalogue \#202436), TAS-102 (MedKoo, catalogue \#205641), trametinib (MedKoo, catalogue \#201458).

\section{ELISA}

Serum samples and media were collected at various time points following 3D tumoroid culture. The total protein content was determined in each sample using the BSA Protein Assay Kit (ThermoFisher Scientific). The protein levels were normalized and subsequently subjected to ELISA for human CEA (ThermoFisher Scientific) and human CA19-9 (ThermoFisher Scientific). The protein standards, media and serum samples were diluted according to the manufacturer's instructions. The resulting colorimetric reaction was analyzed at $562 \mathrm{~nm}$ using a Beckman Coulter DTX880 Multimode Detector. The levels of protein present in each sample were determined mathematically from the straight-line equation of the standard curve.

\section{Histopathology and immunohistochemistry}

Tumoroids and 3D ALI tumor cultures were fixed in formalin and embedded in paraffin. Cut sections were stained with $\mathrm{H} / \mathrm{E}$. Slide sections were rehydrated and subjected to antigen retrieval in citric acid buffer (1.0 $\mathrm{mM}, \mathrm{pH}$ 6.0). Endogenous peroxidases were blocked with $3 \%$ hydrogen peroxide. The slides were blocked for 30 minutes in 5\% goat serum and subsequently incubated with the primary rabbit antibodies towards CD45 (ab10559, AbCam), CD3 (ThermoFisher Scientific), CEA (A0115, Dako) and CA19-9 (M3517, Dako) at room temperature overnight. Slides were then washed and incubated with secondary peroxidase conjugated antibodies using the ImmPRESSTM HRP Anti-Rabbit/ Mouse IgG (Peroxidase) Polymer Detection Kit (Vector Laboratories). Following washes in PBS, the slides were stained using the 3,3'-diaminobenzidine tetrahydrochloride (DAB) Horseradish Peroxidase (HRP) Substrate Kit, 3,3'-diaminobenzidine (Vector Laboratories). The slides were counterstained using hematoxylin.

\section{Profiling of immune cells in patient blood and tumor tissue}

White blood cells (WBC) and tumor-infiltrating cells were divided into a myeloid staining category (CD80, CD68, CD206, CD16, HLA-DR, CD14, CD33, CD45 and propidium iodide [PI]) and a T-cell staining category (CD329, CD328, CD94, CD16, CD4, CD117, CD56, $\mathrm{CD} 3, \mathrm{CD} 8, \mathrm{CD} 45$ and PI). Stained cells were analyzed on a Beckton-Dickinson (BD) ARIA II flow cytometer with four lasers at 633-, 488-, 405- and 365-nm wavelengths (Cell Sorting Facility, Fox Chase Cancer Center). Data were collected with BD FACS Diva software version 6 and analyzed with Flowjo v9.2 (Tree Star Inc.). Single-cell events were first gated by a forward scatter height versus forward scatter area plot and viable CD45+ cells were then gated by lack of PI staining and analyzed for the surface markers indicated for the myeloid and NK/T-cell category.

\section{Abbreviations}

ALI, air-liquid interface; BMP, bone morphogenic protein; CA19-9, carbohydrate antigen 19-9; 2D, two-dimensional; 3D, three-dimensional; CEA, carcinoembryonic antigen; CA19-9, carbohydrate antigen 19-9; CRC, colorectal cancer; DAB, 3,3'-diaminobenzidine tetrahydrochloride; $\mathrm{H} / \mathrm{E}$, hematoxylin-eosin; HRP, horseradish peroxidase; NSCLC, non-small cell lung cancer; PI, propidium iodide; WBC, white blood cells; TSA, tumor shed antigen.

\section{ACKNOWLEDGMENTS}

This work was supported in part by Morphotek Inc. and by the American Cancer Society. The authors would like to thank Mary P Gilroy, Barbara D Dettore, Kathy Q. Cai and Catherine Renner for support with tissue acquisition, tissue embedding and histopathology. W.S.E-D. is an American Cancer Society Research Professor.

\section{CONFLICTS OF INTEREST}

L.G. and N.C.N. are employees of Morphotek Inc. The remaining authors declare that they have no conflict of interest.

\section{REFERENCES}

1. Dutta D, Heo I, Clevers H. Disease Modeling in Stem Cell-Derived 3D Organoid Systems. Trends in Molecular Medicine. 2017; 23:393-410.

2. Ohta Y, Sato T. Intestinal tumor in a dish. Frontiers in Medicine. 2014; 1:14. 
3. van de Wetering M, Francies HE, Francis JM, Bounova G, Iorio F, Pronk A, van Houdt W, van Gorp J, Taylor-Weiner A, Kester L, McLaren-Douglas A, Blokker J, Jaksani S, et al. Prospective derivation of a living organoid biobank of colorectal cancer patients. Cell. 2015; 161:933-945.

4. Verissimo CS, Overmeer RM, Ponsioen B, Drost J, Mertens S, Verlaan-Klink I, Gerwen BV, van der Ven M, Wetering MV, Egan DA, Bernards R, Clevers H, Bos JL, et al. Targeting mutant RAS in patient-derived colorectal cancer organoids by combinatorial drug screening. eLife. 2016; 5 .

5. Weeber F, van de Wetering M, Hoogstraat M, Dijkstra KK, Krijgsman O, Kuilman T, Gadellaa-van Hooijdonk CG, van der Velden DL, Peeper DS, Cuppen EP, Vries RG, Clevers H, Voest EE. Preserved genetic diversity in organoids cultured from biopsies of human colorectal cancer metastases. Proceedings of the National Academy of Sciences of the United States of America. 2015; 112:1330813311.

6. NCT02436564. In Vitro Models of Liver and Pancreatic Cancer. clinicaltrials.gov. 2015. Available at https:// clinicaltrials.gov/ct2/show/NCT02436564

7. Cristobal A, van den Toorn HW, van de Wetering M, Clevers H, Heck AJ, Mohammed S. Personalized Proteome Profiles of Healthy and Tumor Human Colon Organoids Reveal Both Individual Diversity and Basic Features of Colorectal Cancer. Cell Reports. 2017; 18:263-274.

8. Fumagalli A, Drost J, Suijkerbuijk SJ, van Boxtel R, de Ligt J, Offerhaus GJ, Begthel H, Beerling E, Tan EH, Sansom OJ, Cuppen E, Clevers H, van Rheenen J. Genetic dissection of colorectal cancer progression by orthotopic transplantation of engineered cancer organoids. Proceedings of the National Academy of Sciences of the United States of America. 2017; 114:E2357-E2364.

9. Hanahan D, Coussens LM. Accessories to the crime: functions of cells recruited to the tumor microenvironment. Cancer Cell. 2012; 21:309-322.

10. Mlecnik B, Bindea G, Kirilovsky A, Angell HK, Obenauf AC, Tosolini M, Church SE, Maby P, Vasaturo A, Angelova M, Fredriksen T, Mauger S, Waldner M, et al. The tumor microenvironment and Immunoscore are critical determinants of dissemination to distant metastasis. Science Translational Medicine. 2016; 8:327ra326.

11. Grivennikov SI, Greten FR, Karin M. Immunity, inflammation, and cancer. Cell. 2010; 140:883-899.

12. Pancione M, Giordano G, Remo A, Febbraro A, Sabatino L, Manfrin E, Ceccarelli M, Colantuoni V. Immune escape mechanisms in colorectal cancer pathogenesis and liver metastasis. Journal of Immunology Research. 2014; 2014:686879.

13. Ootani A, Li X, Sangiorgi E, Ho QT, Ueno H, Toda S, Sugihara H, Fujimoto K, Weissman IL, Capecchi MR, Kuo CJ. Sustained in vitro intestinal epithelial culture within a Wnt-dependent stem cell niche. Nature Medicine. 2009; 15:701-706.
14. Li X, Nadauld L, Ootani A, Corney DC, Pai RK, Gevaert O, Cantrell MA, Rack PG, Neal JT, Chan CW, Yeung T, Gong X, Yuan J, et al. Oncogenic transformation of diverse gastrointestinal tissues in primary organoid culture. Nature Medicine. 2014; 20:769-777.

15. Fujii M, Matano M, Nanki K, Sato T. Efficient genetic engineering of human intestinal organoids using electroporation. Nature Protocols. 2015; 10:1474-1485.

16. Drost J, van Jaarsveld RH, Ponsioen B, Zimberlin C, van Boxtel R, Buijs A, Sachs N, Overmeer RM, Offerhaus GJ, Begthel H, Korving J, van de Wetering M, Schwank G, et al. Sequential cancer mutations in cultured human intestinal stem cells. Nature. 2015; 521:43-47.

17. Holdenrieder S. Biomarkers along the continuum of care in lung cancer. Scandinavian Journal of Clinical and Laboratory Investigation Supplementum. 2016; 245:S4045.

18. Chang JC, Kundranda M. Novel Diagnostic and Predictive Biomarkers in Pancreatic Adenocarcinoma. International Journal of Molecular Sciences. 2017; 18:667.

19. Das V, Kalita J, Pal M. Predictive and prognostic biomarkers in colorectal cancer: A systematic review of recent advances and challenges. Biomedicine \& Pharmacotherapy. 2017; 87:8-19.

20. Boj SF, Hwang CI, Baker LA, Chio II, Engle DD, Corbo V, Jager M, Ponz-Sarvise M, Tiriac H, Spector MS, Gracanin A, Oni T, Yu KH, et al. Organoid models of human and mouse ductal pancreatic cancer. Cell. 2015; 160:324-338.

21. Hubert CG, Rivera M, Spangler LC, Wu Q, Mack SC, Prager BC, Couce M, McLendon RE, Sloan AE, Rich JN. A Three-Dimensional Organoid Culture System Derived from Human Glioblastomas Recapitulates the Hypoxic Gradients and Cancer Stem Cell Heterogeneity of Tumors Found In Vivo. Cancer Research. 2016; 76:2465-2477.

22. Karthaus WR, Iaquinta PJ, Drost J, Gracanin A, van Boxtel R, Wongvipat J, Dowling CM, Gao D, Begthel H, Sachs N, Vries RG, Cuppen E, Chen Y, et al. Identification of multipotent luminal progenitor cells in human prostate organoid cultures. Cell. 2014; 159:163-175.

23. Gao D, Vela I, Sboner A, Iaquinta PJ, Karthaus WR, Gopalan A, Dowling C, Wanjala JN, Undvall EA, Arora VK, Wongvipat J, Kossai M, Ramazanoglu S, et al. Organoid cultures derived from patients with advanced prostate cancer. Cell. 2014; 159:176-187.

24. McMahon JA, Takada S, Zimmerman LB, Fan CM, Harland RM, McMahon AP. Noggin-mediated antagonism of BMP signaling is required for growth and patterning of the neural tube and somite. Genes \& Development. 1998; 12:1438-1452.

25. Haramis AP, Begthel $\mathrm{H}$, van den Born M, van Es J, Jonkheer S, Offerhaus GJ, Clevers H. De novo crypt formation and juvenile polyposis on BMP inhibition in mouse intestine. Science. 2004; 303:1684-1686.

26. Hardwick JC, Van Den Brink GR, Bleuming SA, Ballester 
I, Van Den Brande JM, Keller JJ, Offerhaus GJ, Van Deventer SJ, Peppelenbosch MP. Bone morphogenetic protein 2 is expressed by, and acts upon, mature epithelial cells in the colon. Gastroenterology. 2004; 126:111-121.

27. He XC, Zhang J, Tong WG, Tawfik O, Ross J, Scoville DH, Tian Q, Zeng X, He X, Wiedemann LM, Mishina Y, Li L. BMP signaling inhibits intestinal stem cell self-renewal through suppression of Wnt-beta-catenin signaling. Nature Genetics. 2004; 36:1117-1121.

28. Kosinski C, Li VS, Chan AS, Zhang J, Ho C, Tsui WY, Chan TL, Mifflin RC, Powell DW, Yuen ST, Leung SY, Chen X. Gene expression patterns of human colon tops and basal crypts and BMP antagonists as intestinal stem cell niche factors. Proceedings of the National Academy of Sciences of the United States of America. 2007; 104:1541815423.

29. Qi Z, Li Y, Zhao B, Xu C, Liu Y, Li H, Zhang B, Wang X, Yang X, Xie W, Li B, Han JJ, Chen YG. BMP restricts stemness of intestinal Lgr5+ stem cells by directly suppressing their signature genes. Nature Communications. 2017; 8:13824.

30. Losi L, Bouzourene H, Benhattar J. Loss of Smad4 expression predicts liver metastasis in human colorectal cancer. Oncology Reports. 2007; 17:1095-1099.
31. Matano M, Date S, Shimokawa M, Takano A, Fujii M, Ohta Y, Watanabe T, Kanai T, Sato T. Modeling colorectal cancer using CRISPR-Cas9-mediated engineering of human intestinal organoids. Nature Medicine. 2015; 21:256-262.

32. Kline JB, Kennedy KR, Albone E, Chao Q, Fernando S, McDonough JM, Rybinski K, Wang W, Somers EB, Schweizer C, Grasso L, Nicolaides NC. Tumor antigen CA125 suppresses antibody-dependent cellular cytotoxicity (ADCC) via direct antibody binding and suppressed Fc receptor engagement. Oncotarget. 2017; 8:52045-52060. https://doi.org/10.18632/oncotarget.19090.

33. Sato T, Stange DE, Ferrante M, Vries RG, Van Es JH, Van den Brink S, Van Houdt WJ, Pronk A, Van Gorp J, Siersema PD, Clevers H. Long-term expansion of epithelial organoids from human colon, adenoma, adenocarcinoma, and Barrett's epithelium. Gastroenterology. 2011; 141:1762-1772.

34. Grivennikov SI, Wang K, Mucida D, Stewart CA, Schnabl B, Jauch D, Taniguchi K, Yu GY, Osterreicher CH, Hung KE, Datz C, Feng Y, Fearon ER, et al. Adenoma-linked barrier defects and microbial products drive IL-23/IL-17mediated tumour growth. Nature. 2012; 491:254-258. 